\title{
Posterolateral rotatory instability of the knee after arthroscopic subtotal lateral meniscectomy: a case report
}

\author{
ERICA BULGHERONI ${ }^{1}$, PAOLO BULGHERONI ${ }^{2}$ \\ ${ }^{1}$ San Raffaele Hospital, Milan, Italy \\ 2 Department of Orthopaedics and Traumatology, University of Insubria, Circolo Hospital and Macchi Foundation, \\ Varese, Italy
}

\begin{abstract}
A 33-year-old Caucasian woman came to our attention complaining of lateral knee pain on the left side, severe external instability associated with varus malalignment, and difficulties in weight-bearing activities. The symptoms had appeared following two lateral meniscectomies on her discoid meniscus, performed elsewhere. The patient was initially submitted to an allograft meniscus transplantation exploiting the unloaded condition of lateral compartment and obtained pain relief. The posterolateral corner was reconstructed in combination with a valgus osteotomy to address the posterolateral rotatory instability. The follow-up assessment at two years after the last surgery showed no symptoms, maintenance of limb alignment and no evidence of joint degeneration.
\end{abstract}

Key Words: discoid meniscus, knee, lateral meniscus, posterolateral corner, rotatory instability.

\section{Introduction}

The posterolateral corner of the knee joint is stabilized by a complex arrangement of structures that include the lateral meniscus (1). The popliteus muscle, the popliteus tendon and its fascicles, and their meniscocapsular attachments are the main stabilizers of the posterolateral region of the knee (2). A lesion of one or

\footnotetext{
Corresponding Author:

Erica Bulgheroni, MD

Via Corridoni 61, 21100 Varese, Italy

Phone: +393490088175

E-mail: ericabulgheroni@hotmail.it
}

more of these structures can be the cause of posterolateral rotatory instability of the knee, with pain and mechanical symptoms at the lateral compartment of the joint (3). Injuries to the posterolateral compartment of the knee are usually reported as a consequence of traumas; however there are no studies in the current literature that describe these injuries as a consequence of surgery.

The present report describes a case of posterolateral rotatory instability of the knee as a possible consequence of lateral meniscectomy, a routinely performed surgical procedure, and the treatment used to address this complex knee disorder.

\section{Case presentation}

A 33-year-old Caucasian woman came to our attention complaining of pain on the lateral side of her left knee, lateral instability whilst standing and walking, and problems in performing weight-bearing activities. The patient reported having undergone two previous lateral arthroscopic partial meniscectomies to the same knee due to the presence of a painful lateral discoid meniscus. The procedures had been performed in another hospital, one and two years previously. From this time of the first surgical procedure, the patient experienced no resolution of the pain and the onset of lateral knee instability.

On clinical examination, the knee showed signs of lateral compartment impairment, with laxity of the posterolateral corner and a varus thrust during walking. A weight-bearing long-standing radiograph with an anteroposterior (AP) view and a Rosenberg view highlighted the presence of 10 degrees of varus malalignment of the affected knee due to capsule ligamentous 
laxity (Fig.1). Magnetic resonance imaging (MRI) confirmed the almost complete absence of the lateral meniscus.

The patient agreed to undergo a complex two-stage sur-

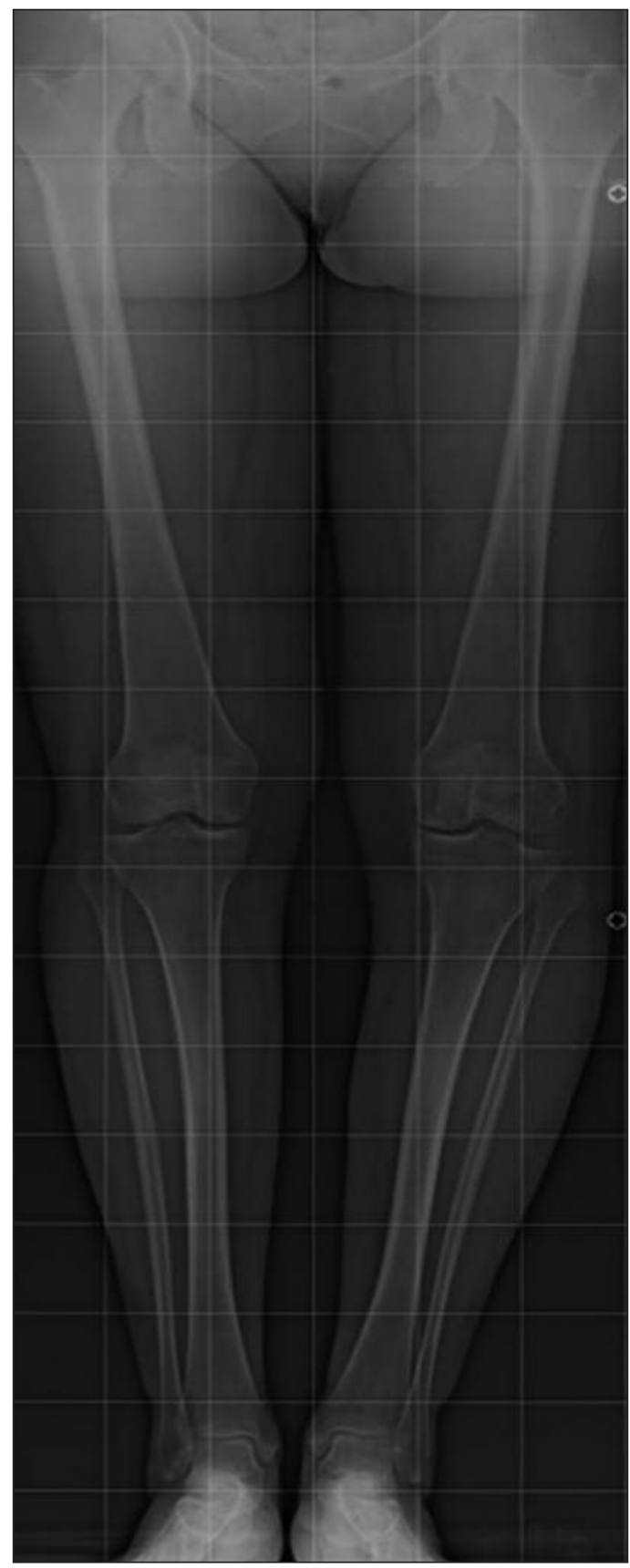

Fig. 1. Weight-bearing long-standing AP radiograph: varus malalignment of the limb due to the insufficiency of the lateral capsuloligamentous structures (arrow). gical treatment consisting of lateral meniscal allograft, ligament reconstruction of the posterolateral corner and opening-wedge valgus osteotomy, undertaken with the aim of resolving the pain and instability. Therefore, as the first stage of the treatment, the patient underwent a meniscal allograft transplantation of the lateral meniscus (Fig. 2). Four months after the surgery, she was pain free and had regained complete range of motion (ROM). Clinical examination and MRI performed at six months showed good recovery despite the persistence of signs and symptoms of posterolateral instability. The patient then underwent reconstruction of the posterolateral corner according to Larson (4) associated with a medial opening-wedge high tibial osteotomy (Fig. 3). Six months after the latter surgery the patient did not complain of her knee giving way; she showed low quadriceps weakness, mild soreness at the posterolateral aspect of the knee and complete ROM. One year after the second surgical procedure, the muscle and pain symptoms appeared to be improved, while X-rays showed consolidation of the osteotomy. Two years after the second surgery the patient complained only of soreness around the hardware used for the osteotomy; radiographically the alignment was maintained and there were no signs of joint degeneration (Fig.4). The hardware was then removed leading to complete remission of the symptoms.

\section{Discussion}

The patient here described presented a complex disorder of the knee joint, in the genesis of which previous surgical procedures seem to have played an important role.

The discoid meniscus is a rare congenital abnormality of the knee. Currently, surgical treatment, consisting of a partial meniscectomy, is indicated only in symptomatic cases (5). However, in the present case, only a small portion of the meniscus was saved after her second meniscectomy and symptoms related to overload and instability appeared. In order to protect the painful lateral compartment, deprived of its natural shock absorber, we implanted a meniscal allograft, which provided immediate pain relief.

Posterolateral instability might be due to prior surgical 

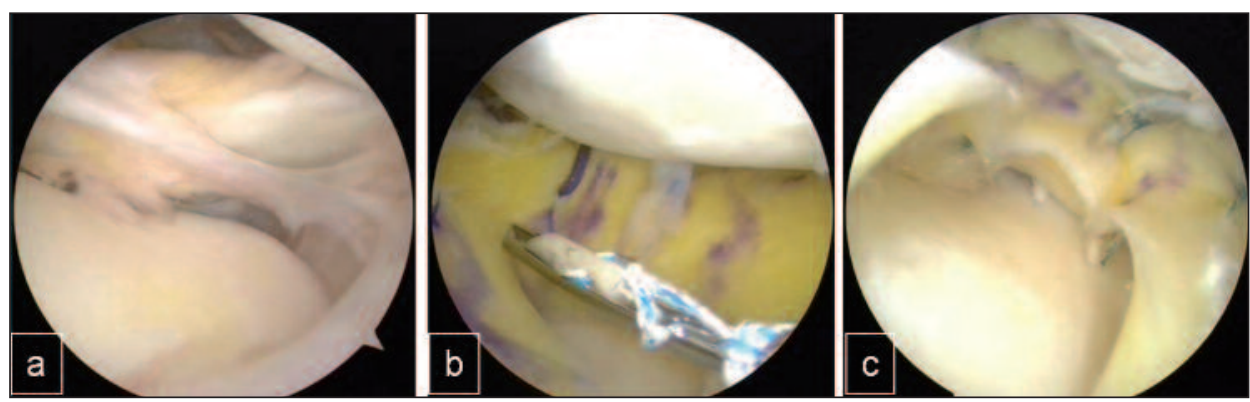

Fig. 2. Arthroscopic images of meniscal allograft transplantation. A: Almost complete absence of the lateral meniscus. B: Fixation of the meniscal allograft. C: Arthroscopic view of the allograft after fixation.

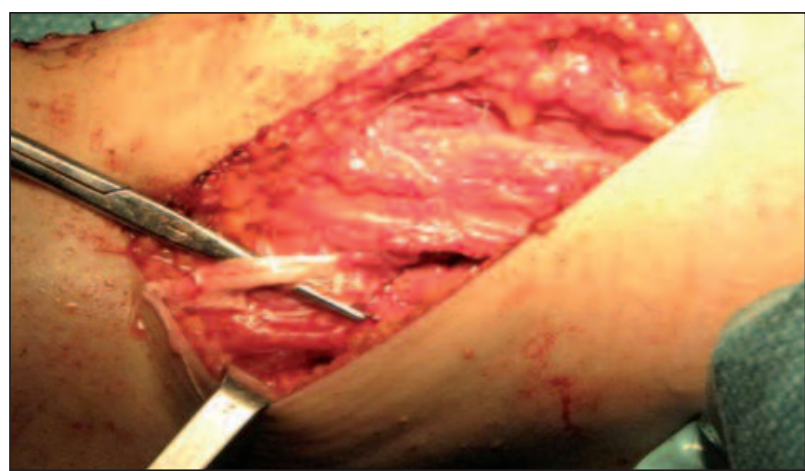

Fig. 3. Reconstruction of the posterolateral corner according to Larson (ref 4).

procedures. The most reasonable hypothesis is that of accidental injuries to the posterolateral anatomical structures connected to the meniscus and responsible for rotatory and lateral stability of the knee. Among these structures, an important role is played by the popliteomeniscal fasciculi. These provide the posterior attachment of the lateral meniscus and are also involved in rotatory stability because of their anchorage to the popliteus tendon, popliteo fibular ligament, posterior capsule, posterior cruciate ligament and popliteus obliquus ligament (6). In fact, the patient did not report any symptoms of instability before the first meniscectomy and the literature suggests that isolated lesions of the lateral meniscus are generally associated with integrity of these fasciculi (2).

Direct visual assessment during open surgery showed integrity of the popliteus tendon and the lateral collateral ligament, although the latter was loose; the other posterolateral capsule ligamentous structures were not clearly identifiable because of the presence of fibrotic

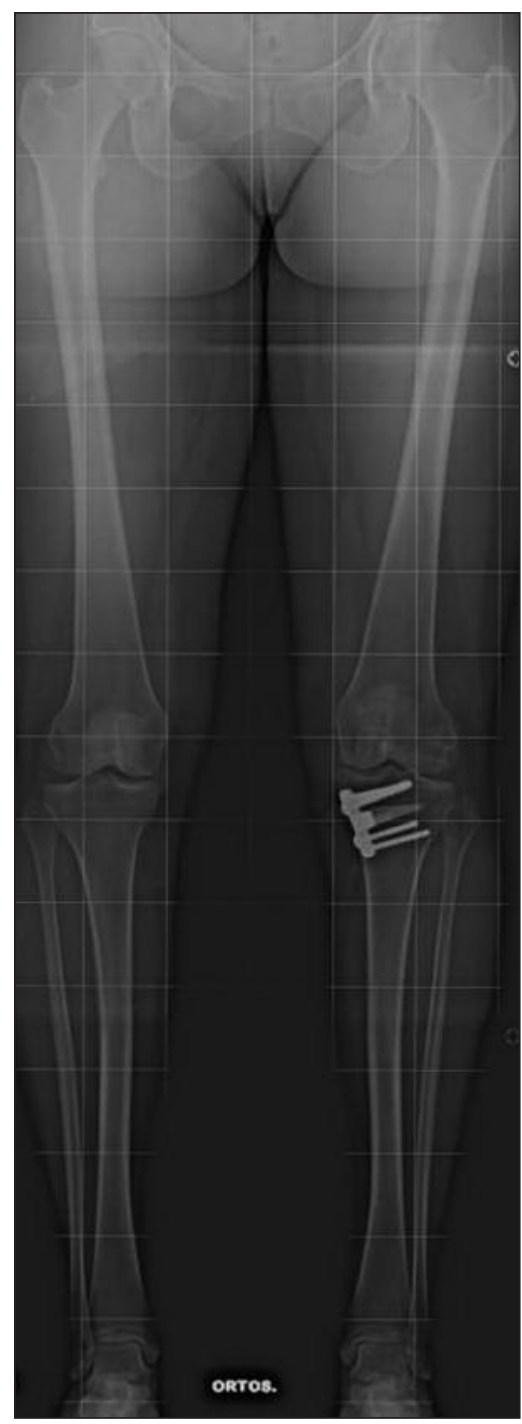

Fig. 4. Weight-bearing long-standing AP radiograph at the two year follow-up: the correction is maintained, the osteotomy is consolidated and the articular surfaces are intact. 


\section{Joints}

scar tissue. The arthroscopic evaluation was not useful for the same reason. Moreover, clinical and MRI evaluations were not able to provide further details, as previously reported in the literature $(3,7)$. The ligament reconstruction was protected by simultaneous correction of the limb alignment, as suggested by the literature (7). Nevertheless, a longer follow-up will be necessary to monitor for recurrence of the symptoms or the onset of degenerative joint disease.

In conclusion, the connections of the lateral meniscus with popliteus tendon and with the posterolateral capsuloligamentous structures are fundamental to ensure stability of the knee joint. They can be damaged during surgical procedures on the lateral meniscus. It is important to be aware of this possible sequela, which requires complex management.
E. Bulgheroni et al.

\section{References}

1. Diamantopoulos A, Tokis A, Tzurbakis M, et al. The posterolateral corner of the knee: evaluation under microsurgical dissection. Arthroscopy. 2005;21:826-833.

2. Staubli HU, Birrer S. The popliteustendon and its fascicles at the popliteal hiatus: gross anatomy and functional arthroscopic evaluation with and without anterior cruciate ligament deficiency. Arthroscopy. 1990;6:209-220.

3. LaPrade RF, Konowalchuk BK. Popliteomeniscal fascicle tears causing symptomatic lateral compartment knee pain. Diagnosis by the figure- 4 test and treatment by open repair. Am J Sports Med. 2005;33:1231-1236.

4. Larson RV. Isometry of the lateral collateral and popliteo fibular ligaments and techniques for reconstruction using a free semitendinosus tendon graft. Oper Tech Sports Med. 2001;9:84-90.

5. Atay OA, Doral MN, Leblebicioğlu G, et al. Management of discoid lateral meniscus tears: observations in 34 knees. Arthroscopy. 2003;19:346-352.

6. Peduto AJ, Nguyen A, Trudell DJ, et al. Popliteomeniscal fascicles: anatomic considerations using $\mathrm{MR}$ arthrography in cadavers. AJR Am J Roentgenol. 2008;190:442-448.

7. Savarese E, Bisicchia S, Romeo R, et al. Role of high tibial osteotomy in chronic injuries of posterior cruciate ligament and posterolateral corner. J Orthop Traumatol. 2011;12:1-17. 